\title{
Low-temperature crystal structure, specific heat, and dielectric properties of lithium tetraborate $\mathrm{Li}_{2} \mathrm{~B}_{4} \mathrm{O}_{7}$
}

\author{
A. Senyshyn, ${ }^{1, a)}$ B. Schwarz, ${ }^{2}$ T. Lorenz, ${ }^{3}$ V. T. Adamiv, ${ }^{4}$ Ya. V. Burak, ${ }^{4}$ J. Banys ${ }^{5}$ \\ R. Grigalaitis, ${ }^{5}$ L. Vasylechko, ${ }^{6}$ H. Ehrenberg, ${ }^{2}$ and H. Fuess ${ }^{1}$ \\ ${ }^{1}$ Institute for Material Science, Darmstadt University of Technology, Petersenstrasse 23, D-64287 \\ Darmstadt, Germany \\ ${ }^{2}$ Leibniz-Institute IFW Dresden, Institute for Complex Materials, P.O. Box 270116, D-01171 Dresden, \\ Germany \\ ${ }^{3}$ II. Physikalisches Institut, Universität zu Köln, Zülpicher Strasse 77, D-50937 Köln, Germany \\ ${ }^{4}$ Institute of Physical Optics, 23 Dragomanov St., 79005 Lviv, Ukraine \\ ${ }^{5}$ Physics Faculty, Vilnius University, Sauletekio 9/3, 10222 Vilnius, Lithuania \\ ${ }^{6}$ Lviv Polytechnic National University, 12 Bandera St., 79013 Lviv, Ukraine
}

(Received 7 June 2010; accepted 20 September 2010; published online 11 November 2010)

Coherent neutron powder diffraction experiments were carried out together with specific heat, dilatometry, and dielectric spectroscopy studies on $\mathrm{Li}_{2} \mathrm{~B}_{4} \mathrm{O}_{7}$ enriched with ${ }^{11} \mathrm{~B}$ isotope to $99.3 \%$ at low temperatures. Neither traces of phase transformations nor discontinuous changes in physical properties were observed. Negative thermal expansion, anomalous thermal behavior of selected interatomic distances/angles, isotropic displacement parameters on specific sites as well as dielectric constant were discussed in terms of dynamic lithium disorder. (C) 2010 American Institute of Physics. [doi:10.1063/1.3504244]

\section{INTRODUCTION}

A variety of borates with different stoichiometries exhibit unique optical characteristics among known nonlinear optical materials, as many such crystals display $\chi^{2}$ and $\chi^{3}$ nonlinear susceptibilities, short cut-off wavelengths thus extending their application range to the deep ultraviolet range of spectrum and an outstanding resistance to laser and nuclear damage. Over long time lithium tetraborate, a member of the very rich borate family of crystals, attracted strong interest due to its exceptional optical properties. For example $\mathrm{Li}_{2} \mathrm{~B}_{4} \mathrm{O}_{7}$ has possible applications as a substrate material for bulk and surface acoustic wave (SAW) devices, ${ }^{1,2}$ as a frequency-conversion material for second-fifth harmonic generation from a high-power Nd:YAG laser, ${ }^{3-5}$ as a highpower ultraviolet light source based on second harmonic generation and sum frequency generation of the visible laser radiation, ${ }^{6,7} \mathrm{Ag}$-doped or $\mathrm{Cu}$-doped $\mathrm{Li}_{2} \mathrm{~B}_{4} \mathrm{O}_{7}$ crystals are used as thermoluminescence dosimeters, ${ }^{8,9}$ as neutron detectors, ${ }^{10}$ etc.

Lithium tetraborate is a broadband dielectric with $E_{8}$ $=7.57 \mathrm{eV}$ and a transmission range of $165-6000 \mathrm{~nm},{ }^{11,12}$ where the boron-oxygen framework mainly contributes to the formation of levels in the upper region of the valence band. ${ }^{13} \mathrm{Li}_{2} \mathrm{~B}_{4} \mathrm{O}_{7}$ shows photoluminescence, when excited with hard quanta ${ }^{12}$ and rather high radiation resistance (approximately $\left.40 \mathrm{GW} / \mathrm{cm}^{2}\right) .{ }^{4}$

According to Ref. 14 the lithium tetraborate crystals have a Mohs hardness of 6 (quartz is 7), a low density $\left(2.45 \mathrm{~g} / \mathrm{cm}^{3}\right)$, acoustic velocities slightly faster than in $\mathrm{LiNbO}_{3}$ and $\mathrm{LiTaO}_{3}$, a SAW reflectivity per stripe five times higher than in $\mathrm{LiNbO}_{3}, \mathrm{LiTaO}_{3}$, or quartz which suggests miniaturization of SAW devices. Lithium tetraborate melts

${ }^{a)}$ Electronic mail: anatoliy.senyshyn@gmail.com. congruently at $1188 \mathrm{~K}^{15}$ and depending on quenching rate the material can be obtained either in crystalline or glassy form. As this work deals only with crystalline lithium tetraborate its amorphous modification will not be considered.

Large single crystals of perfect quality (up to $4 \mathrm{in}$. in diameter) can be grown either by the Czochralski ${ }^{16}$ or Bridgman ${ }^{17}$ method. The structure of $\mathrm{Li}_{2} \mathrm{~B}_{4} \mathrm{O}_{7}$ is unique, i.e., it crystallizes in its own structure type, which was first reported by Krogh-Moe in $1962^{18}$ and since then numerous studies of the crystal structure at different conditions and applying various methods of structure determination and sources of radiation. For example a structure model from Ref. 18 was improved by the authors of Refs. 19 and 20. Mathews et $a .^{21}$ reported structural studies in the temperature range $298 \leq \mathrm{T} \leq 1061 \mathrm{~K}$, authors of Refs. 22 and 23 presented combined powder and $\mathrm{x}$-ray single crystal diffraction studies in a broad temperature range from 123 to $923 \mathrm{~K}$, in Ref. 24 the crystal structure of $\mathrm{Li}_{2} \mathrm{~B}_{4} \mathrm{O}_{7}$ was studied using high-resolution synchrotron powder diffraction in the temperature range $12-298 \mathrm{~K}$.

The studies mentioned above all report the commensurate $I 4_{1} c d$ space group as well as an anomaly of the $c$ lattice parameters below $\mathrm{T} \leq 400 \mathrm{~K}$ for $\mathrm{Li}_{2} \mathrm{~B}_{4} \mathrm{O}_{7}$. In Refs. 25-28 the occurrence of incommensurate modulation in z-direction of tetragonal lattice was reported, where transition to incommensurate structure was induced by periodic temperature variations. At the same time temperature resolved experiments with nuclear magnetic resonance on ${ }^{7} \mathrm{Li}$ and ${ }^{11} \mathrm{~B}$ (Ref. 29) revealed no anomalous behavior of quadruple interactions. Temperature resolved (80-300 K) studies of Bragg reflection with $(00 l)(l=12)$ index on samples undergoing different temperature cycling did not show any evidences for incommensurate modulation in [001] direction. ${ }^{30}$

Complementary to the discussion of incommensurate 
modulations in $\mathrm{Li}_{2} \mathrm{~B}_{4} \mathrm{O}_{7}$, plenty of reports indicating unusual behavior of $\mathrm{Li}_{2} \mathrm{~B}_{4} \mathrm{O}_{7}$ in low-temperature and hightemperature ranges exist. In the current work we will focus on the low temperature properties of lithium tetraborate. Thus pronounced anomalies in the specific heat were observed at 126.2 and $214 \mathrm{~K}$ (Ref. 31) as well as at $238 \mathrm{~K}^{32}$ Unusual temperature behavior of sound velocities and attenuation of sound was observed at 75,125 , and $215 \mathrm{~K}$ in Ref. 33. Dilatometric studies performed in the temperature range $80-250 \mathrm{~K}$ indicate the occurrence of numerous phase transitions. ${ }^{34}$ Anomalies in the Raman spectra were observed at $235 \mathrm{~K}$ by Burak and Moroz, ${ }^{35}$ at $95,185,220$, and $283 \mathrm{~K}$ in Ref. 36 and again at 75,125 , and $215 \mathrm{~K}$ by Sehery and Somerford. ${ }^{33}$ New lines emerged in $\mathrm{A}_{1}(\mathrm{LO})+\mathrm{B}_{1}$ Raman spectra upon cooling from 300 to $156 \mathrm{~K}$ in Ref. 37 . The temperature dependence of the heat conductivity coefficient undergoes a well defined minimum at approximately 238 $\mathrm{K}^{32}$

At the same time experimental studies exist showing no evidence for any anomalies or phase transitions at low temperatures. ${ }^{29,38-42}$ The existing discrepancy between the experimental results of different authors engaged us to perform systematic low-temperature structural studies of lithium tetraborate. For materials containing light elements the neutron scattering methods are known to be more accurate, but to our knowledge no neutron scattering experiments on $\mathrm{Li}_{2} \mathrm{~B}_{4} \mathrm{O}_{7}$ were reported in the literature so far. This fact might be related to the large absorption cross section of natural boron and need for the isotope substitution. Therefore here we present experimental results obtained on $\mathrm{Li}_{2}{ }^{11} \mathrm{~B}_{4} \mathrm{O}_{7}$ using neutron powder diffraction, specific heat, dilatometry, and dielectric spectroscopy.

\section{EXPERIMENTAL DETAILS}

A single crystal of $\mathrm{Li}_{2} \mathrm{~B}_{4} \mathrm{O}_{7}$ with $11 \mathrm{~B}$ :10B isotope ratio 99.6:0.4\% was grown by the Czochralski method in Pt crucible on air at the Institute of Physical Optics (Lviv, Ukraine).

For powder diffraction studies a part of the single crystalline ingot was crushed, ground and homogenized in size. Short check with $\mathrm{x}$-ray powder diffraction reveals only Bragg reflections consistent with $\mathrm{Li}_{2} \mathrm{~B}_{4} \mathrm{O}_{7}$ structure type. ${ }^{18}$ Elastic coherent neutron scattering experiments were performed on the high-resolution powder diffractometer SPODI at the research reactor FRM-II (Garching, Germany). ${ }^{43}$ Monochromatic neutrons $(\lambda=1.5482 \AA)$ were obtained at a $155^{\circ}$ take-off angle using the (551) reflection of a verticallyfocused composite Ge monochromator. The vertical positionsensitive multidetector ( $300 \mathrm{~mm}$ effective height) consisting of $80{ }^{3} \mathrm{He}$ tubes and covering an angular range of $160^{\circ} 2 \theta$ was used for data collection. Measurements were performed in Debye-Scherrer geometry. The powder sample (approximately $2 \mathrm{~cm}^{3}$ in volume) was filled into a thin-wall $(0.15$ $\mathrm{mm}$ ) vanadium can of $14 \mathrm{~mm}$ in diameter and then mounted in the top-loading closed-cycle refrigerator. Helium 4 was used as a heat transmitter. The instantaneous temperature was measured using two thin film resistance cryogenic temperature sensors Cernox and controlled by a temperature controller from LakeShore. Two dimensional powder diffraction data were collected at fixed temperatures in the range of 3.4-268 K upon heating and then corrected for geometrical abberations and curvature of Debye-Scherrer rings.

Alternatively to a neutron powder diffraction experiment thermal expansion of single crystalline $\mathrm{Li}_{2} \mathrm{~B}_{4} \mathrm{O}_{7}$ was directly measured. A single crystalline oriented plate with dimensions approximately $3 \times 3 \times 4 \mathrm{~mm}^{3}$, where the longest edge corresponds to the [001] direction, and shorter ones to [100] and [010], respectively, was mounted in a capacitance dilatometer inserted in a He 4 gas-flow cryostat. Thermal expansion studies were carried out in the broad temperature range from 2 to $300 \mathrm{~K}$.

For heat capacity measurements a single crystalline flat piece was mounted onto the sample holder platform with a small amount of Apiezon $\mathrm{N}$ grease, serving as thermal contact. The calorimeter (PPMS, Quantum Design) uses a relaxation method. The heat capacity of the sample holder together with grease were determined in a separate run and subtracted from the total measured heat capacity. The uncertainty in the molar heat capacity $c_{p}(T)$ has been found to be about $1 \%$ between 2 and $50 \mathrm{~K}$ and increases to $1.5 \%$ at 320 $\mathrm{K}$.

For the dielectric spectroscopy the platelet like crystals with surface parallel to (100) and (001) were used. All measurements were performed in a direction perpendicular to the layers. The complex dielectric permittivity $\mathrm{e}^{*}$ was measured using the HP4284A capacitance bridge in the frequency range $20 \mathrm{~Hz}-1 \mathrm{MHz}$. In the frequency region from $1 \mathrm{MHz}$ to $3 \mathrm{GHz}$ measurements were performed by a coaxial dielectric spectrometer with vector network analyzer Agilent 8714ET. All measurements have been performed on cooling with a controlled temperature rate $0.25 \mathrm{~K} / \mathrm{min}$. Silver paste has been used for contacting.

Treatment of powder diffraction data was carried out using Rietveld technique implemented into the software package FULLPROF. ${ }^{44}$ The peak profile shape was described by a pseudo-Voigt function. The background of the diffraction pattern was fitted using a linear interpolation between selected data points in nonoverlapping regions. The scale factor, lattice parameter, fractional coordinates of atoms, and their isotropic displacement parameters, zero angular shift, profile shape parameters, and half width (Caglioti) parameters were varied during the fitting.

\section{RESULTS AND DISCUSSION}

\section{A. Lattice parameters and microstructure}

Inspection of the obtained neutron diffraction data at different temperatures revealed the presence of reflections consistent with $\mathrm{Li}_{2} \mathrm{~B}_{4} \mathrm{O}_{7}$-type of structure only, thus confirming isostructurality of $\mathrm{Li}_{2} \mathrm{~B}_{4} \mathrm{O}_{7}$ in the temperature range 3.4-300 $\mathrm{K}$. The best Rietveld fit to the powder diffraction pattern collected at $3.4 \mathrm{~K}$ was obtained with parameters listed in Table I.

Lattice parameters $a$ and $c$ of tetragonal $\mathrm{Li}_{2} \mathrm{~B}_{4} \mathrm{O}_{7}$ depend on temperature nonlinearly and smoothly. No traces of anomalies, like spikes, humps, peaks, etc., were observed on thermal dependencies of lattice dimensions [Figs. 1(a) and 
TABLE I. Experimental structural parameters of $\mathrm{Li}_{2} \mathrm{~B}_{4} \mathrm{O}_{7}$ at $T=3.4 \mathrm{~K}$. The space group is $I{ }_{1} c d$ (No. 110). The structural data were modeled for $\mathrm{Li}, \mathrm{B} 1$, $\mathrm{B} 2, \mathrm{O} 1, \mathrm{O} 2$, and $\mathrm{O} 3$ ions occupying $16 b[x, y, z]$ sites, $\mathrm{O} 4$ occupies the site $8 a[0,0, z]$ with origin $z$ fixed to zero. Numbers in parentheses give statistical deviations in the last significant digit.

\begin{tabular}{|c|c|c|c|c|}
\hline \multirow{2}{*}{$\begin{array}{l}a=9.45444(6)(\AA) \\
\begin{array}{l}\text { Atom } \\
\text { site }\end{array}\end{array}$} & \multicolumn{3}{|c|}{$c=10.32360(7)(\AA)$} & \multirow{2}{*}{$\begin{array}{c}V=922.79(2)\left(\AA^{3}\right) \\
B_{i s o} \\
\left(\AA^{2}\right)\end{array}$} \\
\hline & $x / a$ & $y / b$ & $z / c$ & \\
\hline $\mathrm{Li}$ & $0.1523(4)$ & $0.1647(4)$ & $0.8476(4)$ & $0.35(6)$ \\
\hline B1 & $0.1687(1)$ & $0.0870(1)$ & $0.1993(2)$ & $0.18(2)$ \\
\hline B2 & $0.9461(1)$ & $0.1126(1)$ & $0.0812(2)$ & $0.08(2)$ \\
\hline O1 & $0.2829(1)$ & 0.1392(1) & $0.2651(2)$ & $0.06(2)$ \\
\hline $\mathrm{O} 2$ & $0.0673(1)$ & $0.1777(1)$ & $0.1565(2)$ & $0.24(2)$ \\
\hline $\mathrm{O} 3$ & $0.1570(1)$ & $0.9431(1)$ & $0.1804(2)$ & $0.20(2)$ \\
\hline $\mathrm{O} 4$ & 0 & 0 & 0 & $0.08(3)$ \\
\hline$R_{p}: 4.15 \%$ & \multicolumn{2}{|c|}{$R_{w p}: 5.02 \%$} & $R_{\text {exp }}: 2.01 \%$ & $\chi^{2}: 6.21$ \\
\hline
\end{tabular}

1(b)]. However, a strong anisotropy of their thermal evolution occurs: to some extent the $a$ lattice parameter displays an "usual" increasing behavior, while $c$ lattice parameter nonlinearly decreases upon heating. The observed anisotropy of thermal expansion causes a well-defined minimum on the thermal dependence of cell volume around $130 \mathrm{~K}$ [Fig. 1(c)].

Such an anisotropy of thermal expansion was already reported in the literature, e.g., in Ref. 23 it is defined that $c$ lattice parameter shows a minima on its temperature evolution around $500 \mathrm{~K}$. Recent high-resolution powder diffraction studies using synchrotron radiation ${ }^{24}$ performed on $\mathrm{Li}_{2} \mathrm{~B}_{4} \mathrm{O}_{7}$ with natural isotope composition showed lattice contraction in [001] direction of the lattice, but did not reveal any extrema on thermal dependence of cell volume.

These inconsistencies can be explained by different radiation sources used. As it has been already mentioned in Ref. 45 in case of complex structures the accuracy of lattice parameter determination from high-resolution neutron powder diffraction is higher than those using x-ray diffraction or even synchrotron radiation. Improved accuracy of neutron data can be explained by the better counting statistics at high $2 \theta$ angles and the improved average of crystallites over the irradiated sample due to much bigger sample amount. Another reason for observed discrepancies, which cannot be excluded is the isotope effect.

In addition to the pronounced anisotropy of thermal expansion an unusual behavior was observed in temperature evolution of peak widths. Check of profile shape parameters yields their minor but systematic dependence on temperature, which would anticipate the development of strain/size effects at low temperatures. The deconvolution of observed reflection broadening with instrumental resolution function was performed and further analysis of Williamson-Hall plots reveals that the average size of crystallites remains constant in the whole temperature region, while the microstrain in $\mathrm{Li}_{2} \mathrm{~B}_{4} \mathrm{O}_{7}$ is tempting to develop upon cooling. The determination of complete strain ellipsoid failed due to a relatively weak strain magnitude. Thus the best stable fit was achieved with the anisotropic microstructural model based on [001] axial strain vector.

The obtained maximum (upper limit) strain, related by a constant factor to the root-mean-square-strain and corresponding to $1 / 4$ of the apparent strain defined by Stokes and
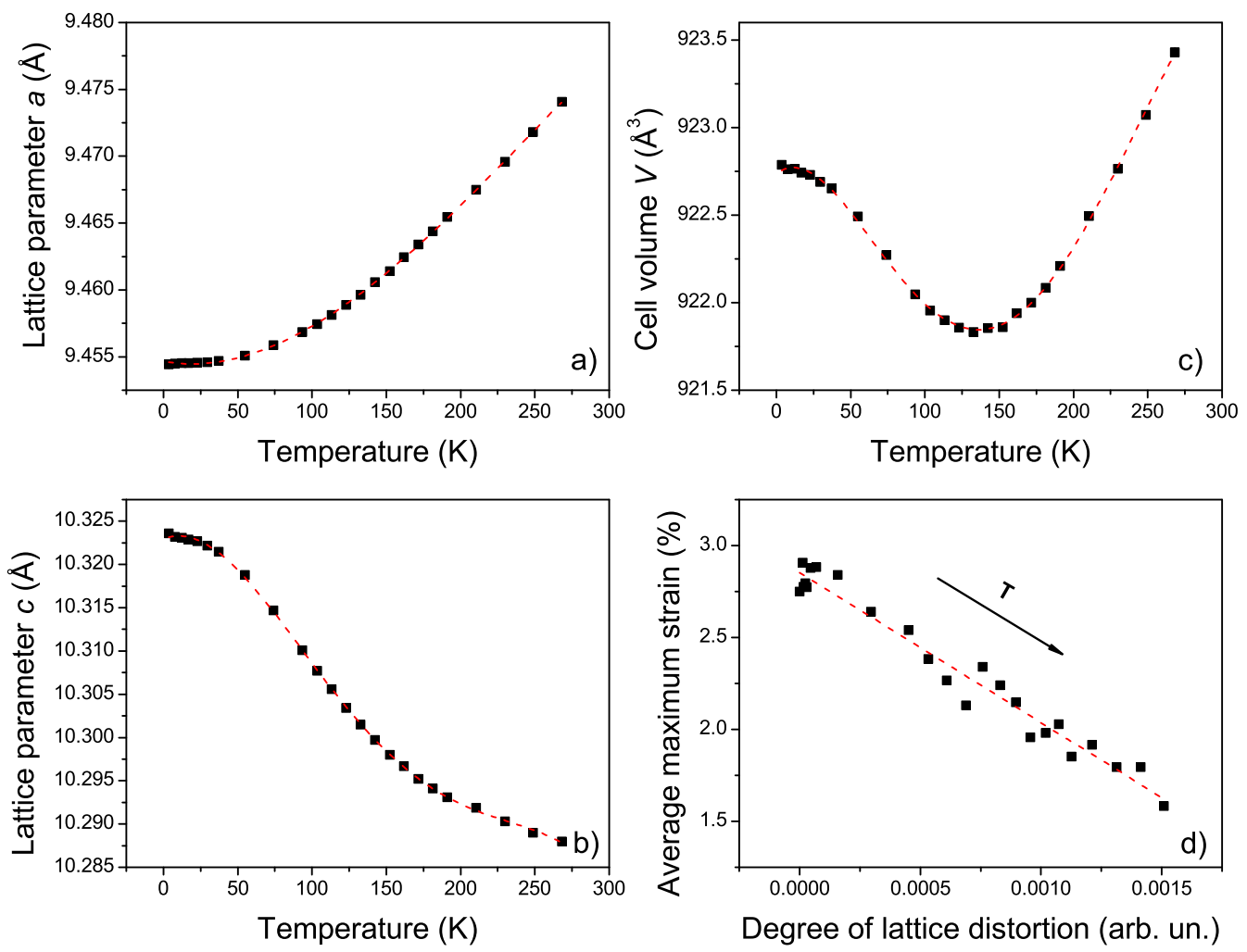

FIG. 1. (Color online) Temperature dependencies of lattice parameters (a) and (b), cell volume (c), and average maximum strain vs degree of lattice distortion (d). Dashed lines are shown as guide for eyes. 


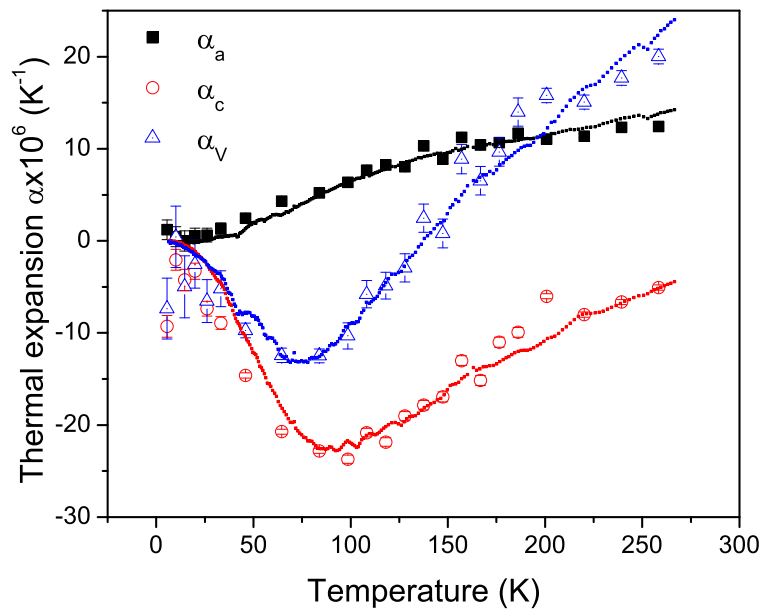

FIG. 2. (Color online) Temperature dependencies of linear and volumetric TECs. Solid points denote data from neutron powder diffraction and dotsdilatometric studies.

Wilson ${ }^{46}$ increases upon cooling. The observed evolution of microstrain can be related to the anisotropy of thermal expansion via lattice distortion $x=x+\Delta$ and can be described in the crystallographic basis of the initial lattice by the Lagrangian tensor of finite deformations. ${ }^{47}$ Then the degree of lattice distortion can be defined as a spontaneous strain (square root of the sum of squared eigenvalues of strain tensor) divided by 3. As Fig. 1(d) shows, both average maximum strain and the degree of lattice distortion depend linearly on each other thus indicating the significant anisotropy of thermal expansion to be the possible reason for broadening of reflections at low temperatures.

\section{B. Thermal expansion and specific heat}

For uniaxial crystals the thermal expansion tensor $\alpha_{k l}$ has two independent components $\alpha_{a}=\alpha_{11}$ and $\alpha_{c}=\alpha_{33}$, where $\alpha_{a}$ and $\alpha_{c}$ denote thermal expansion coefficient (TEC) either in $a$ - or $c$-directions, respectively. The volumetric TEC $\alpha_{V}$ then is just a sum of the respective anisotropic TECs: $2 \alpha_{a}$ $+\alpha_{c}$. Cell sizes (lattice parameters and cell volume) $l(T)$ are related to the TEC $\alpha_{l}(T)$ through the $V-T$ equation of state as

$$
l(T)=l\left(T_{0}\right) \exp \left\{\int_{T_{0}}^{T} \alpha_{l}(x) d x\right\},
$$

where $T_{0}$ stands for the initial temperature $(3.4 \mathrm{~K})$. And conversely TEC $\alpha_{l}(T)$ can be determined from cell sizes (lattice parameters and cell volume) $l(T)$ by simple derivation

$$
\alpha_{l}(T)=\frac{1}{l(T)} \frac{d l}{d T} .
$$

The temperature dependencies of components of the thermal expansion tensor for $\mathrm{Li}_{2} \mathrm{~B}_{4} \mathrm{O}_{7}$ are shown in Fig. 2, where TECs calculated from cell dimensions are shown by large points, directly measured TECs from dilatometry study are presented as dots. Both data sets are in fair agreement thus indicating similar thermal expansion of single crystal and powder species. The $\alpha_{a}$ TEC is nonlinearly increasing upon heating, whereas the trends of $\alpha_{c}$ and $\alpha_{V}$ curves indicates a

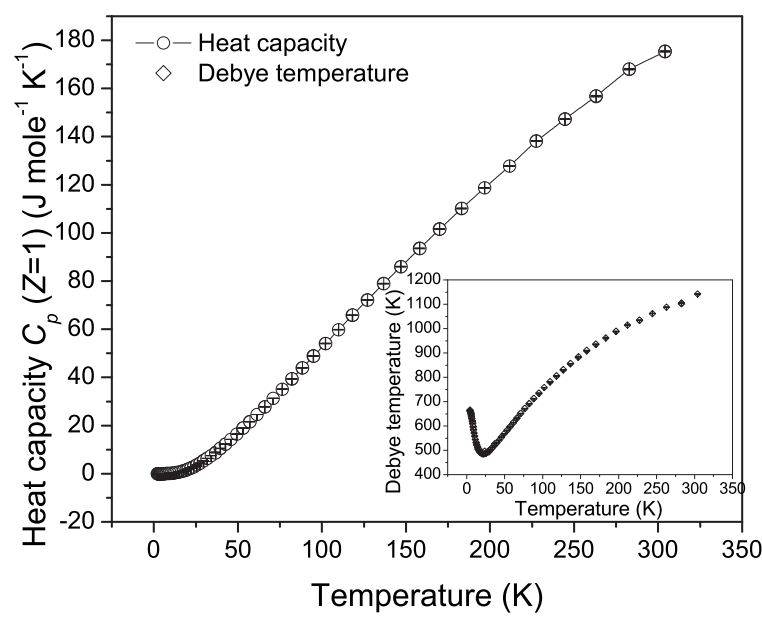

FIG. 3. Temperature dependence of isobaric heat capacity in $\mathrm{Li}_{2} \mathrm{~B}_{4} \mathrm{O}_{7}$ (thermal dependence of Debye temperature is shown in inset).

minimum around $90 \mathrm{~K}$ and $75 \mathrm{~K}$, respectively. Note that $\alpha_{c}$ is negative in the whole temperature range below room temperature (RT) and might approach zero around $470 \mathrm{~K}^{23}$

Within quasiharmonic approximation the volumetric TEC can be expressed via fundamental parameters of solids, such as the Grüneisen parameter, $\rho$ is the density of the material, the isothermal and adiabatic bulk modules $K_{T}$ and $K_{S}$ and the isochoric and isobaric heat capacities $C_{V}$ or $C_{P}$

$$
\alpha_{V}=\frac{\gamma_{V} \rho C_{V}}{K_{T}}=\frac{\gamma_{V} \rho C_{P}}{K_{S}}
$$

For an uniaxial crystal this approach can be extended by using transverse and longitudinal projections of the Grüneisen parameter and full elastic constant tensor, which allowed to consider anisotropic expansion. ${ }^{48}$ However, the applicability of quasiharmonic approximation to lithium tetraborate is quite doubtful. Previously reported pronounced anisotropy of Raman shifts in longitudinal and transverse directions together with anomalies in thermal dependencies of elastic tensor components ${ }^{39}$ as well as the observed development of microstrain indirectly point out the relevance of the $(\partial \omega \backslash \partial T)_{V} U$ term in general definition for TEC, i.e., phonon frequencies will probably show quite different response on volume than on temperature.

Nevertheless measurement of isobaric specific heat (which gives the major contribution to the TEC) were performed and results are shown in Fig. 3. Measurements upon cooling/heating revealed neither hysteresis nor discontinuous changes in the thermal dependence of specific heat, thus confirming isostructurality of $\mathrm{Li}_{2} \mathrm{~B}_{4} \mathrm{O}_{7}$ in the whole temperature range considered. The specific heat of lithium tetraborate is quite low, e.g., even at room temperature $C_{p}$ is far away from its saturated value (Dulong-Petit limit) of $324.266(\mathrm{~J} / \mathrm{mole}$ $\times \mathrm{K})$.

From the lattice term (which is believed to be solely present in measured $c_{p}(T)$ ) the equivalent Debye temperature $\theta_{D}(T)$ can be calculated. ${ }^{49}$ Obtained dependence (inset in Fig. 3) has been found highly nonlinear, thus unambiguously indicated that $\mathrm{Li}_{2} \mathrm{~B}_{4} \mathrm{O}_{7}$ can be poorly described within Debye theory at elevated temperatures. Similar to 


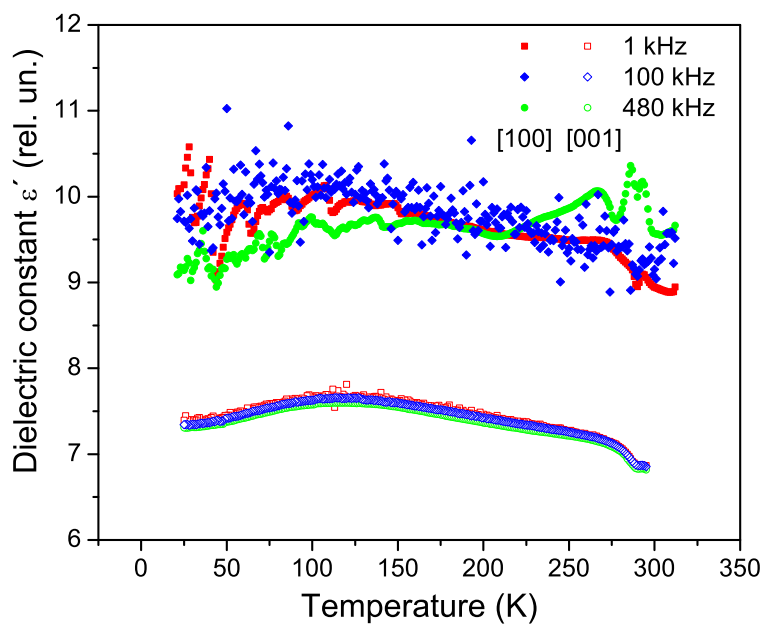

FIG. 4. (Color online) Temperature and frequency dependencies of the dielectric permittivity $\epsilon$ in the [100] (open points) and [001] (solid points) direction of tetragonal lattice.

perovskite-type $\mathrm{e}^{50-53}$ and sheelite-type ${ }^{54,55}$ materials one can define possible reasons for deviations from Debye law: complicated structure and atomic coordination, gaps in phonon spectrum (regions of contribution to the total phonon density of states, ${ }^{56}$ which are well separated in energy), numerous stretching modes, etc.

\section{Dielectric spectroscopy}

Significant anisotropy of structural properties will result in an anisotropy of dielectric properties. Considering the specific crystal structure, Abrahams ${ }^{57}$ reported that lithium tetraborate is most likely not ferroelectric but piezoelectric and pyroelectric. Nevertheless the dielectric properties of $\mathrm{Li}_{2} \mathrm{~B}_{4} \mathrm{O}_{7}$ were studied by different authors and results of these studies are sometimes in disagreement.

At room temperature lithium tetraborate is characterized by a relatively low dielectric constant $\epsilon$, but reasonably high $\tan \sigma$ values. Usually thermal and frequency dependencies of $\epsilon$ in [100] and [110] direction show Debye-like behavior and $\epsilon_{a}$ is nearly temperature independent over broad temperature range $4-700 \mathrm{~K}$, whereas different kinds of anomalies were observed in $c$-direction at low and high temperatures. Most of the authors who studied dielectric properties of $\mathrm{Li}_{2} \mathrm{~B}_{4} \mathrm{O}_{7}$ (Refs. 58-65) noticed an increase in $\epsilon_{c}$ supplemented by pronounced frequency dispersion just around room temperature, where temperatures of such anomalies vary from approximately. $200 \mathrm{~K}$ in Ref. 61 to approximately $450 \mathrm{~K}$ in Ref. 63. Furthermore, lithium tetraborate showed an unusual dynamic hysteresis loop in $P-E$ measurements, ${ }^{64}$ which is strongly correlated with dielectric constant and conductivity.

We performed dielectric measurements on the current sample. The temperature dependencies of the dielectric constants $\epsilon_{a}$ and $\epsilon_{c}$ in the low-temperature region are shown in Fig. 4.

As one can see in $a$-direction of tetragonal lattice the dielectric permittivity does not respond on frequency and remains almost constant at 7.4 in the whole temperature range studied, which is in agreement with the observation of other authors. A similar behavior but much more noisy, has been noticed for $\epsilon_{c}=9.7$. Approaching room temperature neither frequency dispersion nor systematic increase in $\epsilon$, reported in the literature, was seen, therefore, we expect it to occur above room temperature.

Deeper understanding of a noisy $\epsilon$ behavior in $c$-direction of the tetragonal lattice can be achieved from extended analysis of crystal structure, bonding, and thermal vibrations.

\section{Bond-length analysis}

It is well known that the crystal structure of lithium tetraborate can be described as space arrangement of a tetraborate group $\left[\mathrm{B}_{4} \mathrm{O}_{7}\right]^{2-}$ and lithium. A tetraborate group is formed by two $\mathrm{BO}_{4}$ tetrahedra and two $\mathrm{BO}_{3}$ triangles [see Fig. 5(a)] and they are linked via shared $\mathrm{O} 1$ atoms to form interpenetrating networks where cavities in one are filled in by the other. A set of structural parameters defining the behavior of a tetraborate group has to be considered, where the most obvious ones are boron-oxygen bond lengths in triangles and tetrahedra. Thermal evolution of respective interatomic distances are shown in Figs. 5(b) and 5(c), respectively.

Despite the fact that the mean $\langle\mathrm{B}-\mathrm{O}\rangle$ distances remain almost unaffected by temperature, the thermal behavior of the independent $\mathrm{B}-\mathrm{O}$ bond lengths in the tetraborate group is more complicated. In the triangular coordination three unique boron-oxygen distances with completely different stiffness to temperature exist, e.g., the B1-O1 bond contracts upon heating, the $\mathrm{B} 1-\mathrm{O} 2$ one displays thermal expansion, whereas $\mathrm{B} 1-\mathrm{O} 3$ remains almost independent on temperature. A similar feature occurs in the tetragonal oxygen coordination of boron: the B2-O1 and B2-O3 distances show only a weak response on heating with extrema around $100 \mathrm{~K}$, the B2-O4 bond length weakly increases upon heating. The B2-O2 interatomic distance is almost constant below $100 \mathrm{~K}$ but at further temperature increase it contracts by approximately $0.015 \AA$.

A temperature stability of the tetraborate group $\left[\mathrm{B}_{4} \mathrm{O}_{7}\right]^{2-}$ can be illustrated by the $\mathrm{B}-\mathrm{O} 4-\mathrm{B}$ interatomic angles. The B1a-O4-B1b interatomic angle displays minor thermal dependence, i.e., smoothly decreases from $82.2(1)^{\circ}$ at $3.4 \mathrm{~K}$ to $81.9(1)^{\circ}$ at approximately $280 \mathrm{~K}$. At temperatures above approximately $110 \mathrm{~K}$ the angle $\mathrm{B} 2 \mathrm{a}-\mathrm{O} 4-\mathrm{B} 2 \mathrm{~b}$ shows temperature-independent behavior $\left[108.4(1)^{\circ}\right]$, whereas at lower temperatures it starts to grow up upon cooling and reaches $109.2(1)^{\circ}$ at $3.4 \mathrm{~K}$.

Therefore the tetraborate group $\left[\mathrm{B}_{4} \mathrm{O}_{7}\right]^{2-}$ can be considered as relatively stiff to temperature changes, which rules out the distortion and tilting of the tetraborate group $\left[\mathrm{B}_{4} \mathrm{O}_{7}\right]^{2-}$ as the reason for anomalies in thermal expansion of lithium tetraborate. Nevertheless observed rearrangements of boron inside oxygen polyhedra, together with little changes in intertetrahedral angle cannot allow to suppose it being a rigid body.

Arrangement of the tetraborate groups $\left[\mathrm{B}_{4} \mathrm{O}_{7}\right]^{2-}$ form channels along the $\mathrm{c}$ tetragonal axis, which are occupied by chains of $\mathrm{Li}$ and observed overall shift in the tetraborate group $\left[\mathrm{B}_{4} \mathrm{O}_{7}\right]^{2-}$ might be somehow related to rearrangement 

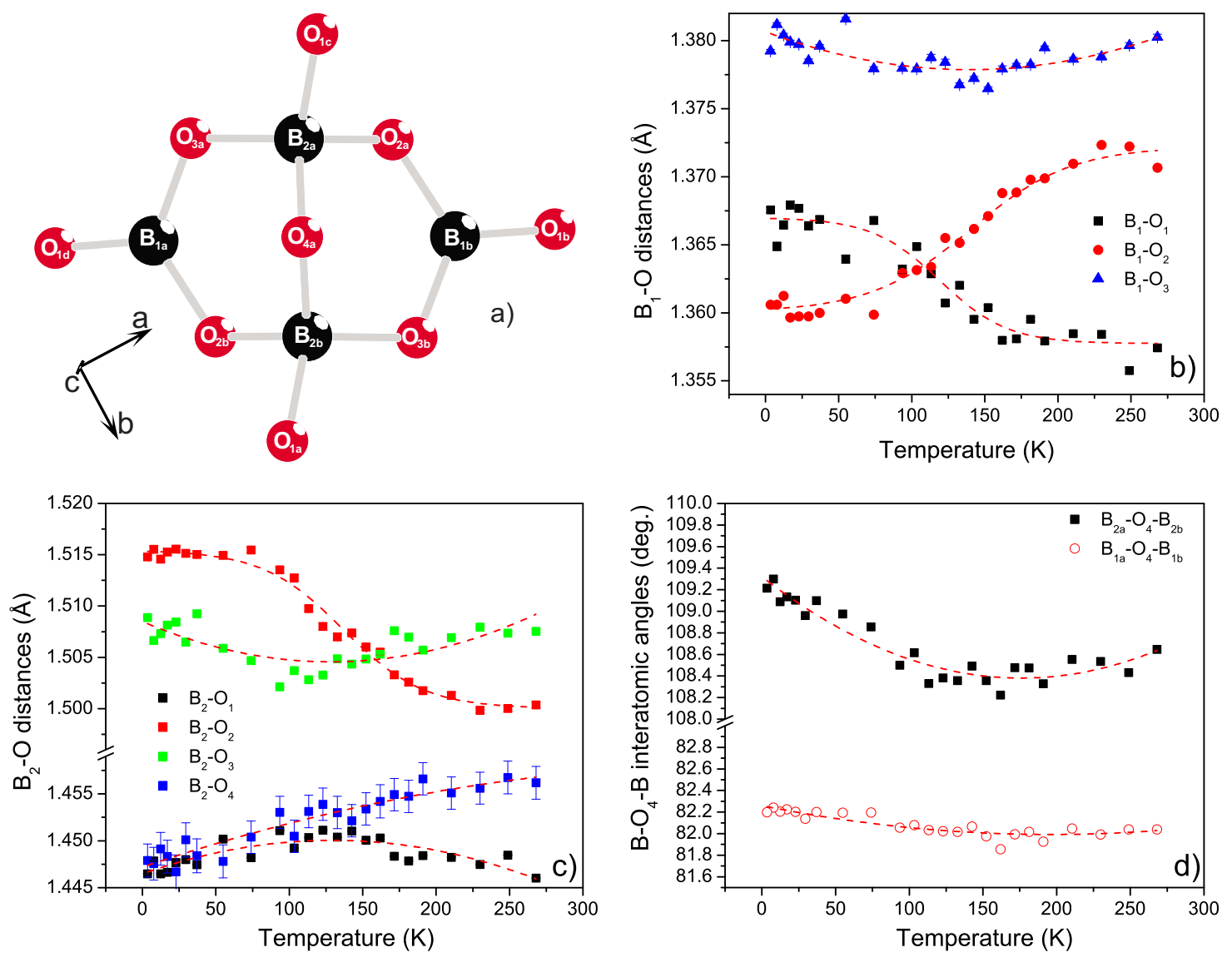

FIG. 5. (Color online) Tetraborate group $\left[\mathrm{B}_{4} \mathrm{O}_{7}\right]^{2-}$ (a) and thermal dependencies of selected interatomic distances (b) and (c) and angles (d).

of lithium ions. The $\mathrm{Li}-\mathrm{O}$ polyhedron can be presented as a distorted tetrahedron built on $\mathrm{O} 1, \mathrm{O} 2, \mathrm{O} 3 \mathrm{a}$, and $\mathrm{O} 3 \mathrm{~b}$, whereas at higher temperatures it can be better defined as a distorted bipyramid ${ }^{23}$ built on $\mathrm{O} 1, \mathrm{O} 2, \mathrm{O} 3 \mathrm{a}, \mathrm{O} 3 \mathrm{~b}$, and $\mathrm{O} 4$. The Li-O1 interatomic distance almost linearly increases upon heating from 2.130(2) $\AA$ at $3.4 \mathrm{~K}$ to $2.183(4) \AA$ at 268 $\mathrm{K}$. The $\mathrm{Li}-\mathrm{O} 2$ bond length displays linear increase as well [1.950(1) $\AA$ at $3.4 \mathrm{~K}$ and $1.967(2)$ at $268 \mathrm{~K}]$ but with smaller magnitude of thermal elongation. Thermal behavior of $\mathrm{Li}-\mathrm{O} 3$ was found nonlinear and can be fairly simulated by Boltzmann sigmoid, e.g., Li-O3a distance at temperatures below $100 \mathrm{~K}$ is almost temperature independent 2.090(2) $\AA$, then it shows linear increase to 2.102(2) $\AA$ and from $180 \mathrm{~K}$ it again becomes insensitive to temperature. A similar feature, but with opposite sign, was observed for temperature dependence of $\mathrm{Li}-\mathrm{O} 3 \mathrm{~b}$ distance as well: at the temperatures below $100 \mathrm{~K}$ and above $180 \mathrm{~K}$ it showed temperature independent

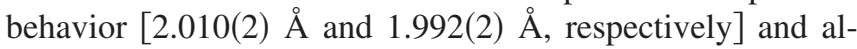
most linear decrease in between these two temperatures. $\mathrm{Li}-\mathrm{O} 4$ bond length, which is already beyond the first coordination sphere of lithium in $\mathrm{Li}_{2} \mathrm{~B}_{4} \mathrm{O}_{7}$, was found to be shortened upon heating in the whole temperature range considered [2.640(2) $\AA$ at $3.4 \mathrm{~K}$ to $2.604(3) \AA$ at $268 \mathrm{~K}]$.

Temperature induced changes in position of lithium have been found more significant than those of other species in the structure, e.g., at low temperatures (below $100 \mathrm{~K}$ ) its position in the lattice is almost constant but at further heating lithium starts to move thus being shifted at $268 \mathrm{~K}$ from the respective position at $3.4 \mathrm{~K}$ by approximately $0.35 \AA$. Lithium is forming discontinuous chains around $4_{1}$ symmetry axis and an example of such a chain is shown in Fig. 6. Interatomic distances $\mathrm{Li}-\mathrm{Li}$ as well as $\mathrm{Li}-\mathrm{Li}-\mathrm{Li}$ interatomic angles are equal for every segment of chain. Lattice parameter c can be defined in terms of this bond length relationships as

$$
c=4 d_{\mathrm{LiLi}} \sqrt{1-2 \cos ^{2}\left(\frac{\alpha_{\mathrm{LiLiLi}}}{2}\right)},
$$

where $d_{\mathrm{LiLi}}$ corresponds to $\mathrm{Li}-\mathrm{Li}$ distance and $\alpha_{\mathrm{LiLiLi}}$ is the $\mathrm{Li}-\mathrm{Li}-\mathrm{Li}$ interatomic angle within the Li-chain. As one can see from Eq. (4) the negative thermal expansion in this direction can be realized either by contraction of $\mathrm{Li}-\mathrm{Li}$ distance or by clockwise rotation of the Li-chain along a $4_{1}$ symmetry axis, which is reflected in reduction in $\mathrm{Li}-\mathrm{Li}-\mathrm{Li}$ angles. Analysis of thermal evolution of $\mathrm{Li}-\mathrm{Li}$ bonds within the chain revealed their linear increase upon heating [3.4 K: 3.110(7) $\AA$ and $268 \mathrm{~K}: 3.131(7) \AA]$, whereas magnitude of $\mathrm{Li}-\mathrm{Li}-\mathrm{Li}$ interatomic angles has been found linearly decreasing upon heating $\left[3.4 \mathrm{~K}: 133.5(2)^{\circ}\right.$ and $\left.268 \mathrm{~K}: 132.4(2)^{\circ}\right]$. The atomic arrangement realized in the $\mathrm{Li}_{2} \mathrm{~B}_{4} \mathrm{O}_{7}$ structure type is much more sensitive to changes in $\mathrm{Li}-\mathrm{Li}-\mathrm{Li}$ angle

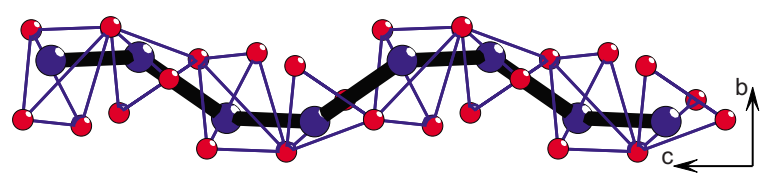

FIG. 6. (Color online) Chain of $\mathrm{LiO}_{4}$ polyhedra formed along $c$-axis in lithium tetraborate. View along [001] (left) and [100] (right) directions. 


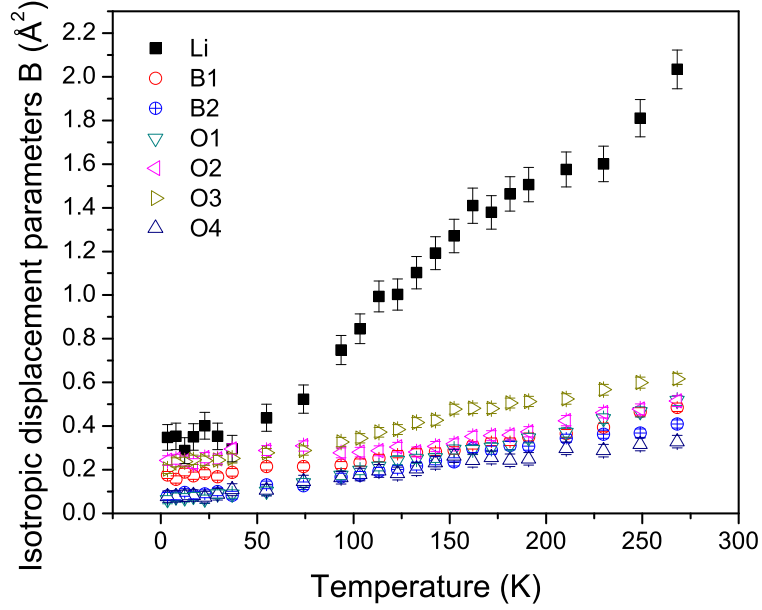

FIG. 7. (Color online) Isotropic displacement parameters in $\mathrm{Li}_{2} \mathrm{~B}_{4} \mathrm{O}_{7}$.

than in $\mathrm{Li}-\mathrm{Li}$ bond lengths, therefore, observed reduction in $\alpha_{\mathrm{LiLiLi}}$ is the reason for anisotropic lattice contraction in lithium tetraborate. ${ }^{22}$

Nevertheless reasons for such kind of anomalous behavior for lithium in $\mathrm{Li}_{2} \mathrm{~B}_{4} \mathrm{O}_{7}$ have to be considered in detail. It is well known that lithium often tends to occupy disordered sites in crystal structures. For sake of completeness it makes sense to extend the discussion to a short consideration of thermal vibrations in $\mathrm{Li}_{2} \mathrm{~B}_{4} \mathrm{O}_{7}$.

\section{E. Displacement parameters}

Approaching room temperature lithium in $\mathrm{Li}_{2} \mathrm{~B}_{4} \mathrm{O}_{7}$ shows relatively high isotropic displacement parameter $\left[B_{\text {iso }}=2.22(7) \AA^{2}\right]$, while the isotropic displacement parameters for other constituents do not exceed $0.7 \AA^{2}$. Thermal evolution of isotropic displacement parameters is shown in Fig. 7.

It is evident that the magnitude of lithium vibrations changes drastically upon cooling from 268 to $3.4 \mathrm{~K}$ in comparison to other atomic sites, which can be attributed to a dynamic disorder. Below $40 \mathrm{~K}$ displacement parameters for all species are almost temperature independent (within uncertainty limits). At $3.4 \mathrm{~K}$ the $B_{\text {iso }}^{\mathrm{Li}}$ was determined as $0.35(6) \AA^{2}$, while for other atomic sites it was found as small as $\quad B_{i s o}^{\mathrm{B} 1}=0.18(2) \AA^{2}, \quad B_{i s o}^{\mathrm{B} 2}=0.08(2) \AA^{2}, \quad B_{\text {iso }}^{\mathrm{O} 1}$ $=0.06(2) \AA^{2}, B_{\text {iso }}^{\mathrm{O} 2}=0.24(2) \AA^{2}, B_{\text {iso }}^{\mathrm{O} 3}=0.20(2) \AA^{2}$, and $B_{\text {iso }}^{\mathrm{O} 4}$ $=0.08(3) \AA^{2}$. Bearing in mind the similarity in atomic weight of lithium, boron, and oxygen this difference may indicate some amount of static disorder on lithium site. Upon heating this difference smoothly increases, which can be related to a development of dynamical disorder on lithium site.

At $3.4 \mathrm{~K}$ the isotropic displacement parameter for B2 site has been found less in comparison with the respective thermal vibration of $\mathrm{B} 1$. The slope of thermal development for $B_{i s o}^{\mathrm{B} 1}$ and $B_{i s o}^{\mathrm{B} 2}$ were found to be very similar, therefore, one can conclude a static disorder on triangularly coordinated B1 site. Disorder phenomena on lithium site were supplemented by anomalous behavior of isotropic displacement parameters for oxygen which is reflected in the redistribution of isotropic displacement parameters in the temperature range $90 \mathrm{~K}$ $\geq T \geq 200 \mathrm{~K}$. Below $90 \mathrm{~K}$ the isotropic displacement param- eters follow the relation: $\left[B_{i s o}^{\mathrm{O} 2} \approx B_{i s o}^{\mathrm{O} 3}\right] \geq\left[B_{\text {iso }}^{\mathrm{O} 1} \approx B_{\text {iso }}^{\mathrm{O} 4}\right]$, whereas at temperatures above $200 \mathrm{~K}$ this inequality reads as $B_{i s o}^{\mathrm{O} 3}$ $\geq\left[B_{\text {iso }}^{\mathrm{O} 1} \approx B_{\text {iso }}^{\mathrm{O} 2}\right] \geq B_{\text {iso }}^{\mathrm{O} 4}$. Observed temperature ranges are in good agreement with these where anomalies in boronoxygen and lithium-oxygen coordinations were shown previously.

\section{CONCLUSIONS}

Coherent neutron powder diffraction experiments were carried out together with specific heat, dilatometry, and dielectric spectroscopy studies at low temperatures on $\mathrm{Li}_{2} \mathrm{~B}_{4} \mathrm{O}_{7}$ enriched with ${ }^{11} \mathrm{~B}$ isotope to $99.3 \%$. Neither traces of phase transformations nor discontinuous changes in physical properties were observed. Thermal lattice contraction in [001] direction of tetragonal lattice was confirmed and found to be supplemented by anomalous behavior of selected interatomic distances and interatomic angles as well as of isotropic displacement parameters. Observed anomalies can be attributed to a dynamical lithium disorder in lithium tetraborate which actively develops at temperatures $100 \mathrm{~K}>T>200 \mathrm{~K}$. The following scenario for negative thermal expansion in $\mathrm{Li}_{2} \mathrm{~B}_{4} \mathrm{O}_{7}$ is proposed: according to Ref. 56 lithium vibrations are dominating to low-frequency phonon branches in the phonon spectrum, i.e., low-energy phonon modes are mostly formed by Li movements. A dynamical disorder on Li site causes clockwise rotations of Li-chains along $4_{1}$ symmetry axis, which leads to a reduction in LiLiLi interatomic angle within Li-chain, thus causing such modes to obey negative mode Grüneisen parameters in special directions of the Brillouin zone.

Following Ref. 23 the anisotropic displacement ellipsoid of lithium is elongated toward $\mathrm{Li}-\mathrm{O} 4$ bond. Therefore observed scattering of values for dielectric permittivities in z-direction of $\mathrm{Li}_{2} \mathrm{~B}_{4} \mathrm{O}_{7}$ as well as their weak frequency dispersion can be associated with preferential direction of disorder.

This assumption is supported by conductivity measurements on $\mathrm{Li}_{2} \mathrm{~B}_{4} \mathrm{O}_{7}$ reported in Refs. 63 and 65, where rather high conductivity (strongly ionic by its nature) has been found along the polar axis. In perpendicular direction the conductivity was about three orders of magnitude lower indicating its quasi-one-dimensional character. ${ }^{37}$ Mobility of ions has been found decreasing as temperature reduces and below $150 \mathrm{~K}$ the mobility closely approaches zero, ${ }^{64}$ which might be related to the suppression of dynamical disorder. However, the ionic conductivity of lithium tetraborate is still several orders of magnitude smaller than the conductivity of typical superionic conductors. Observed quasi-onedimensional character of conductivity can be directly correlated with the crystal structure of lithium tetraborate and can be related to observed scattering of dielectric data in [001]direction.

It seems that physical properties (incl. occurrence of phase transformations and modulation of crystal structure) of lithium tetraborate are highly dependent on the crystal qualitylmethod of growth, which explains controversial results of different authors. For example in Ref. 61 authors performed dielectric spectroscopy on two different 
samples, ${ }^{4,66}$ where in a sample for a nonlinear optical device a small cusp at approximately $220 \mathrm{~K}$ was evident on thermal dependence of $\epsilon_{c}$, whereas no effect was visible on a sample prepared for a SAW device. The origin of such discrepancies is not yet well understood but studies are in progress.

The current work was performed very close to the limit of accuracy for applied experimental methodologies. More precise knowledge on structural behavior, phonon subsystem and disorder in lithium tetraborate can be obtained from single crystal neutron diffraction experiments, measurements of phonon dispersion, nuclear magnetic resonance studies at low temperatures and from either semiclassical or ab initio simulations beyond ground state approximation.

The paraelectric phase in $\mathrm{Li}_{2} \mathrm{~B}_{4} \mathrm{O}_{7}$ is unknown. Authors in Refs. 37 and 65 observed the decrease in second harmonic generation signal at high temperatures thus suggesting the possibility of the phase transition to a nonpolar phase. This fact is indirectly confirmed by an anomalous behavior of different physical properties at elevated temperatures reported by different authors. To our knowledge the hightemperature structural experiments up to the melting point are not reported in the literature so far but they are highly envisaged to prove if polar phase is solely present in crystalline lithium tetraborate in the whole range of its existence.

\section{ACKNOWLEDGMENTS}

This work was partially supported by the German Federal Ministry of Education and Research (BMBF Project No. 03FU7DAR).

${ }^{1}$ R. W. Whatmore, N. M. Shorrocks, C. Ohara, F. W. Ainger, and I. M. Young, Electron. Lett. 17, 11 (1981).

${ }^{2}$ Y. Ebata, H. Suzuki, S. Matsumura, and K. Fukuda, Jpn. J. Appl. Phys., Part 1 22, 160 (1983).

${ }^{3}$ G. C. Bhar, A. K. Chaudhary, P. Kumbhakar, and A. M. Rudra, J. Phys. D: Appl. Phys. 34, 360 (2001).

${ }^{4}$ R. Komatsu, T. Sugawara, K. Sassa, N. Sarukura, Z. Liu, S. Izumida, Y. Segawa, S. Uda, T. Fukuda, and K. Yamanouchi, Appl. Phys. Lett. 70, 3492 (1997).

${ }^{5}$ S. Das, C. Ghosh, and S. Gangopadhyay, Appl. Phys. Lett. 91, 141109 (2007).

${ }^{6}$ R. Komatsu, T. Sugawara, N. Watanabe, S. Uda, and V. Petrov, Rev. Laser Eng. 27, 541 (1999).

${ }^{7}$ V. Petrov, F. Rotermund, F. Noack, R. Komatsu, T. Sugawara, and S. Uda, J. Appl. Phys. 84, 5887 (1998).

${ }^{8} \mathrm{~K}$. Mahesh and P. S. Weng, Thermoluminescence in Solids and its Applications (Nuclear Technology, Ahford, 1989).

${ }^{9}$ C. Furetta and P. S. Weng, Operation Thermoluminiscent Dosimetry (World Scientific, London, 1998).

${ }^{10}$ T. Nakamura, M. Katagiri, Y. E. Chen, M. Ukibe, and M. Ohkubo, Nucl. Instrum. Methods Phys. Res. A 559, 766 (2006).

${ }^{11}$ Y. V. Burak, Y. O. Dovgii, and I. V. Kityk, Sov. Phys. Solid State 31, 275 (1989).

${ }^{12}$ O. T. Antonyak, Y. V. Burak, I. T. Lyseiko, N. S. Pidzyrailo, and Z. A. Khapko, Opt. Spectrosc. 61, 550 (1986).

${ }^{13}$ A. Y. Kuznetsov, L. I. Isaenko, A. V. Kruzhalov, I. N. Ogorodnikov, and A. B. Sobolev, Sov. Phys. Solid State 41, 48 (1999).

${ }^{14}$ J. G. Gualtieri, J. A. Kosinski, W. D. Wilber, Y. Lu, S. T. Lin, M. Murray, and W. Ruderman, Proceedings of 1992 IEEE Frequency Control Symposium (Hershey, PA, USA, 1992), pp. 724-731.

${ }^{15}$ M. D. Mathews, A. K. Tyagi, and P. N. Moorthy, Thermochim. Acta 319, 113 (1998).

${ }^{16}$ V. T. Adamiv, Y. V. Burak, I. S. Girnyk, Y. Kasperczyk, I. V. Kityk, and I. M. Teslyuk, J. Funct. Mater. 4, 415 (2007).

${ }^{17}$ J. Xu, S. Fana, and B. Lua, J. Cryst. Growth 264, 260 (2004).

${ }^{18}$ J. Krogh-Moe, Acta Crystallogr. 15, 190 (1962).
${ }^{19}$ J. Krogh-Moe, Acta Crystallogr., Sect. B: Struct. Crystallogr. Cryst. Chem. 24, 179 (1968).

${ }^{20}$ S. F. Radaev, L. A. Muradyan, L. F. Malakhova, Y. A. Burak, and V. I. Simonov, Sov. Phys. Crystallogr. 34, 842 (1989).

${ }^{21}$ M. D. Mathews, A. K. Tyagi, and P. N. Moorthy, Thermochim. Acta 320, 89 (1998).

${ }^{22}$ N. Sennova and R. Bubnova, J. Shepelev, S. Filatov, and O. Yakovleva, J. Alloys Compd. 428, 290 (2007).

${ }^{23}$ N. Sennova, R. S. Bubnova, G. Cordier, B. Albert, S. K. Filatov, and L. Isaenko, Z. Anorg. Allg. Chem. 634, 2601 (2008).

${ }^{24}$ V. T. Adamiv, Y. V. Burak, and I. M. Teslyuk, J. Alloys Compd. 475, 869 (2009).

${ }^{25}$ V. V. Zaretskiy and Y. V. Burak, JETP Lett. 49, 198 (1989).

${ }^{26}$ V. V. Zaretskiy and Y. V. Burak, Sov. Phys. Solid State 31, 80 (1989).

${ }^{27}$ N. D. Zhigadlo and V. V. Zareckiy, JETP Lett. 49, 498 (1989).

${ }^{28}$ N. D. Zhigadlo, Phys. Status Solidi A 152, 329 (1995).

${ }^{29}$ Y. N. Ivanov, Y. V. Burak, and K. S. Aleksandrov, Sov. Phys. Solid State 32, 3379 (1990).

${ }^{30}$ E. M. Zub, Sov. Phys. Solid State 39, 1297 (1997).

${ }^{31}$ N. P. Tekhanovich, A. U. Sheleg, and Y. V. Burak, Sov. Phys. Solid State 32, 2513 (1990).

${ }^{32}$ A. É. Aliev, V. F. Krivorotov, and P. K. Habibulaev, Sov. Phys. Solid State 39, 1378 (1997).

${ }^{33}$ A. A. Sehery and D. J. Somerford, J. Phys.: Condens. Matter 1, 2279 (1989).

${ }^{34}$ K. Y. Borman and Y. V. Burak, Izv. Akad. Nauk SSSR, Neorg. Mater. 26, 440 (1990) Inorg. Mater. 26, 372 (1990).

${ }^{35}$ Y. V. Burak and I. E. Moroz, Phys. Chem. Glasses 44, 241 (2003).

${ }^{36}$ A. E. Aliev and R. R. Valetov Sov. Phys. Solid State 34, 3061 (1992).

${ }^{37}$ V. S. Gorelik, A. V. Vdovin, and V. N. Moiseenko, J. Russ. Laser Res. 24, 553 (2003)

${ }^{38}$ G. L. Paul and W. Taylor, J. Phys. C 15, 1753 (1982).

${ }^{39}$ H. A. A. Sidek, G. A. Saunders, and B. James, J. Phys. Chem. Solids 51, 457 (1990).

${ }^{40}$ G. I. Malovichko, V. G. Grachev, and A. O. Matkovskii, Sov. Phys. Solid State 33, 1966 (1991).

${ }^{41}$ N. D. Zhigadlo, M. Zhang, and E. K. H. Salje, J. Phys.: Condens. Matter 13, 6551 (2001).

${ }^{42}$ N. D. Baisa, Z. Trybula, V. M. Rizak, I. M. Rizak, and V. M. Holovey, Ukr. Phys. J. 47, 568 (2002).

${ }^{43}$ M. Hoelzel, A. Senyshyn, R. Gilles, H. Boysen, and H. Fuess, Neutron News 18, 23 (2007).

${ }^{44}$ T. Roisnel and J. Rodriguez-Carvajal, Mater. Sci. Forum 378-381, 118 (2001).

${ }^{45}$ A. Senyshyn, D. M. Trots, J. M. Engel, L. Vasylechko, H. Ehrenberg, T. Hansen, M. Berkowski, and H. Fuess, J. Phys.: Condens. Matter 21, 145405 (2009).

${ }^{46}$ A. R. Stokes and A. J. C. Wilson, Proc. Phys. Soc. 56, 174 (1944).

${ }^{47}$ M. Catti, Acta Crystallogr., Sect. A: Found. Crystallogr. 41, 494 (1985).

${ }^{48}$ N. R. Abdulchalikova and A. E. Aliev, Synth. Met. 71, 1929 (1995).

${ }^{49}$ T. H. K. Barron, W. T. Berg, and J. A. Morrison, Proc. R. Soc. London, Ser. A 242, 478 (1957).

${ }^{50}$ A. R. Oganov, J. P. Brodholt, and G. D. Price, Phys. Earth Planet. Inter. 122, 277 (2000).

${ }^{51}$ A. Senyshyn, L. Vasylechko, M. Knapp, U. Bismayer, M. Berkowski, and A. Matkovskii, J. Alloys Compd. 382, 84 (2004).

${ }^{52}$ A. Senyshyn, A. R. Oganov, L. Vasylechko, H. Ehrenberg, U. Bismayer, M. Berkowski, and A. Matkovskii, J. Phys.: Condens. Matter 16, 253 (2004).

${ }^{53}$ A. Senyshyn, H. Ehrenberg, L. Vasylechko, J. D. Gale, and U. Bismayer, J. Phys.: Condens. Matter 17, 6217 (2005).

${ }^{54}$ A. Senyshyn, H. Kraus, V. Mikhailik, and V. Yakovyna, Phys. Rev. B 70, 214306 (2004)

${ }^{55}$ A. Senyshyn, H. Kraus, V. B. Mikhailik, L. Vasylechko, and M. Knapp, Phys. Rev. B 73, 014104 (2006).

${ }^{56}$ V. V. Maslyuk, T. Bredow, and H. Pfnür, Eur. Phys. J. B 42, 461 (2004).

${ }^{57}$ S. C. Abrahams, Acta Crystallogr., Sect. B: Struct. Sci. 52, 790 (1996).

${ }^{58}$ A. S. Bhalla, L. E. Cross, and R. W. Whatmore, Jpn. J. Appl. Phys., Part 1 24, 727 (1985).

${ }^{59}$ M. Maeda, H. Tachi, K. Honda, and I. Suzuki, Jpn. J. Appl. Phys., Part 1 33, 1965 (1994).

${ }^{60}$ A. E. Aliev, A. Sh. Akramov, R. R. Valetov, and P. K. Khabibulaev, Solid State Ionics 46, 197 (1991).

${ }^{61}$ Y. Akishige and R. Komatsu, J. Phys. Soc. Jpn. 73, 1341 (2004). 
${ }^{62}$ J.-W. Cha, H.-R. Jung, C.-S. Kim, J.-S. Kim, H.-H. Baek, and J.-N. Kim, J. Korean Phys. Soc. 27, 303 (1994).

${ }^{63}$ J. S. Kim, J. Phys. Soc. Jpn. 70, 3129 (2001).

${ }^{64}$ C.-S. Kim, B.-E. Jun, H.-H. Baek, D.-J. Kim, Y.-H. Hwang, H.-K. Kim, G S. Jeen, and J. N. Kim, J. Korean Phys. Soc. 42, S1038 (2003).
${ }^{65}$ S. Furusawa, S. Tange, Y. Ishibashi, and K. Miwa, J. Phys. Soc. Jpn. 59, 2532 (1990).

${ }^{66}$ R. Komatsu, T. Sugawara, S. Tsuchiya, S. Uda, K. Sassa, and K. Yamanouchi, Jpn. J. Appl. Phys., Part 1 35, 3180 (1996). 